\title{
Construçáo e Validação da Escala de Avaliação do Debriefing associado à Simulaçáo (EADaS)
}

\author{
Construction and Validation of the Simulation Debriefing Assessment Scale (Escala de \\ Avaliação do Debriefing associado à Simulação - EADaS) \\ Construcción y validación de una Escala para la Evaluación del Debriefing asociado a la \\ Simulación $(\mathrm{EADaS})$
}

Verónica Rita Dias Coutinho*; José Carlos Amado Martins**; Maria de Fátima Carneiro Ribeiro Pereira***

\section{Resumo}

Enquadramento: O debriefing é uma forma estruturada de conduzir os formandos na reflexão sobre a ação, oferecendo uma realidade, através dos seus olhos, do formador e seus pares. Numa simulação, o debriefing é parte fundamental, à qual se deve dedicar tanta atenção como à resolução do cenário. Uma ferramenta que permita avaliar a qualidade do debriefing, na perspetiva do formando, é importante para a investigação e melhoria contínua da qualidade.

Objetivos: Construir e validar uma escala de avaliação do debriefing associado à simulação.

Metodologia: Estudo de investigação metodológica. Foi desenvolvida uma lista de 50 itens, através de revisão da literatura e experiência dos investigadores, na forma de questionário, a 209 estudantes do Curso de Licenciatura em Enfermagem. Resultados: Após análise das respostas, resultou uma escala com 34 itens, com elevada consistência interna (Alpha = 0,899). A análise fatorial sugere divisão em três fatores, todos com elevados valores de Alpha e com significado racional. Conclusão: A versão final da escala apresenta boas propriedades psicométricas, revelando potencial para utilização em investigações futuras.

Palavras-chave: debriefing; simulação; simulação de paciente; estudos de validação.

\begin{abstract}
Background: Debriefing is a structured way for trainees to reflect on action, offering a reality, a way to see through their own eyes, the trainers' and their peers' eyes. Debriefing is an essential aspect of simulation, which should receive as much attention as the resolution of the scenario. A tool to assess the quality of the debriefing from the trainee's perspective is important for research and the continuous quality improvement.

Objectives: To construct and validate a simulation debriefing assessment scale.

Methodology: Methodological research study. A list of 50 items was developed based on the literature review and the researchers' experience, and applied as a questionnaire to 209 students of a Bachelor Degree in Nursing.

Results: After the analysis of the answers, a 34-item scale was designed, with high internal consistency (Alpha $=0.899$ ). The factor analysis suggested a 3-factor solution, all of which with factors showing high Alpha values and rational significance.

Conclusion: The final version of the scale showed good psychometric properties with potential for use in future studies.
\end{abstract}

Keywords: debriefing; simulation; patient simulation; validation studies.

\footnotetext{
*Licenciada em Enfermagem. Pós-Graduada em Enfermagem de Emergência. Especialista em Enfermagem Médico-Cirúrgica. Doutoranda no Curso de Doutoramento em Ciências de Enfermagem - Instituto de Ciências Biomédicas Abel Salazar, Universidade do Porto. Professora Adjunta na Escola Superior de Enfermagem de Coimbra, 3046-851, Coimbra, Portugal [vcoutinho@esenfc.pt]. uperior de Enfermag Morada de correponden cia: Rua Carlos Garrido, n ${ }^{\circ} 6,2^{\circ}$ esq $q^{\circ}-2500-071$ Caldas da Rainha **ós-Graduado em Urgências/Emergências. Mestre em Bioética. Doutor em Ciências de Enfermagem. Enfermeiro Especialista em Enfermagem Médico-Cirúrgica. Coordenador da Unidade Científico Pedagógica de Enfermagem Médico-Cirúrgica. Professor Adjunto na Escola Superior de Enfermagem de Coimbra, Portugal, 3046-851, Coimbra, Portugal [jmartins@esenfc.pt].

*** Licenciada em Ciências da Educação. Doutora em Ciências da Educação. Professora Auxiliar na Faculdade de Psicologia e de Ciências da Educação da Universidade do Porto, 4200-135, Porto, Portugal [fpereira@fpce.up.pt].
}

\section{Resumen}

Marco contextual: El debriefing es una forma estructurada de conducir a los alumnos en la reflexión sobre la acción, ofreciendo una realidad, una manera de verse con sus propios ojos, los del profesor y sus compañeros. En una simulación, el debriefing es la pieza clave a la cual alumnos y profesores deben dedicar tanta atención como a la resolución de la práctica. Tener una herramienta para evaluar la calidad del debriefing en la perspectiva del alumno es importante para la investigación y para la mejora continua de la calidad.

Objetivo: Construcción y validación de una escala para la evaluación del debriefing asociado a la simulación.

Metodología: Investigación metodológica. Se desarrolló una lista de 50 ítems a partir de la revisión de la literatura y la experiencia de los investigadores y se aplicó, en forma de cuestionario, a 209 estudiantes de la Licenciatura en Enfermería.

Resultados: Del análisis de las respuestas resultó una escala con 34 ítems, con una alta consistencia interna (Alpha = 0,899). El análisis factorial sugiere una división en tres factores, todos con altos valores de Alpha y con significado racional. Conclusión: Podemos decir que la versión final de la escala presenta buenas propiedades psicométricas, lo que demuestra su potencial de uso en futuras investigaciones.

Palabras clave: debriefing; simulación; simulación de paciente; estudios de validación

Recebido para publicação em: 02.04 .13

Aceite para publicação em: 28.02 .14 


\section{Introdução}

O debriefing é uma forma estruturada de conduzir os formandos na reflexão sobre a ação, ajudando a consolidar saberes e a mudar comportamentos errados. Numa simulação, o debriefing é parte fundamental. Dispor de uma ferramenta que permita avaliar a qualidade do debriefing, na perspetiva do formando, é importante para a investigação e melhoria contínua da qualidade, não existindo nenhum instrumento em Língua Portuguesa. Assim, pareceunos útil e pertinente a construção de um instrumento que permitisse tal avaliação, pelo que partimos para o presente estudo com o objetivo de construir e validar uma escala de avaliação do debriefing associado à simulação.

\section{Enquadramento}

\section{O debriefing na simulação}

$\mathrm{Na}$ formação dos enfermeiros, as metodologias ativas têm vindo a conquistar terreno. Nelas destaca-se a simulação, um método interativo que permite, não apenas a aprendizagem de habilidades, mas também de competências globais, como a tomada de decisão em situações e ambientes complexos. Através de cenários completos e complexos, em ambiente de simulação, os formandos consolidam os saberes e desenvolvem todo um conjunto de competências que favorece o seu desenvolvimento técnico, relacional e ético (Martins, 2009).

Trata-se de utilizar estratégias educativas que facilitama aprendizagem a partir de situações reais, potenciando o crescimento e confiança dos estudantes (Rodrigues \& Baia, 2012).

Um dos aspetos importantes na simulação é o realismo. Falamos hoje em simulação de altafidelidade ou simulação realística quando associamos um simulador de alta-fidelidade a espaços, materiais e equipamentos reais e tecnologia de som e imagem que permitam o registo áudio e vídeo, e a sua posterior utilização para discussão (Martins et al., 2012). Acrescentam os autores que, não bastam o material e o equipamento, sendo indispensável um cenário bem delineado, com objetivos pedagógicos bem definidos e uma equipa de trabalho, preparada e motivada, terminando a experiência clínica simulada com uma discussão (debriefing) em torno do desempenho dos formandos na resolução do cenário.

O debriefing é referido na literatura como o ponto crucial ou essencial para a aprendizagem, o coração e a alma da simulação (Gaba, Howard, Fish, Smith, \& Sowb, 2001; Baldwin, 2007). É o processo pelo qual formadores e formandos reexaminam a experiência clínica simulada, fomentando o desenvolvimento do raciocínio e consolidação dos saberes, através de processos de aprendizagem reflexiva. A orientação do formador neste processo e a sua centralidade nos objetivos são os pontos centrais para o sucesso (Shinnick, Horwich, \& Steadman, 2011). O debriefing é ainda importante como ferramenta de ajuda aos estudantes, melhorando o domínio afetivo da aprendizagem (Lasater, 2007).

Na simulação, a fase de debriefing é um processo intencional e importante, projetado para coordenar, fortalecer e transferir aprendizagem de um exercício de aprendizagem experiencial (Warrick, Hunsaker, Cook, \& Altman, 1979). O debriefing tem como objetivos centrais: identificar as diferentes perceções e atitudes que ocorrem; relacionar o exercício com a teoria específica ou conteúdos e competências técnicas; transmitir feedback acerca da natureza e da prática durante o cenário; e estabelecer um clima que permita confiança e conforto.

O debriefing pode acontecer pós-simulação ou na simulação, sendo os resultados, para os estudantes, melhores no primeiro caso, pela eficácia na aprendizagem e compreensão do global (Heukelom, Begaz, \& Treat, 2010; Shinnick et al., 2011).

Para além disso, muitos autores recomendam um estilo sem julgamento, não ameaçador, que não se limite a um mero interrogatório (Dreifuerst, 2009).

O debriefing é um processo dinâmico que requer dos participantes e formadores uma participação ativa, devendo ser planeado um período de tempo igual ou superior ao tempo consumido na prática simulada (Gururaja, Yang, Paige, \& Chauvin, 2008).

Ainda assim, há pouca evidência de resultados associados à prática de debriefing, seja por este não ter sido um foco de atenção dos investigadores, seja pela inexistência de instrumentos que permitam a avaliação de tais resultados. No nosso país, o tema é novo, não existindo nenhum instrumento de avaliação. 


\section{Metodologia}

Trata-se de um estudo de investigação metodológica. Começámos por construir uma lista de frases (itens), em torno do conceito central (debriefing), tendo por base a experiência dos investigadores e revisão de literatura sobre a temática. Nesta revisão da literatura, foram centrais as ideias expostas por Jeffries (2007); Kardong-Edgren, Starkweather, e Ward (2008); Campbell e Daley (2009); Baldwin (2007); e Heukelom et al. (2010).

Deste processo resultou uma lista de 50 itens (13 formulados em sentido inverso) que organizámos num formato que permite apenas uma alternativa de resposta, onde o estudante tinha de se posicionar numa escala tipo Likert, de cinco pontos, em que cada ponto representa o nível de concordância do estudante: discordo completamente (1); discordo (2); nem concordo nem discordo (3); concordo (4); e concordo completamente (5).

Procurando utilizar como critérios fundamentais a clareza, a compreensão simples e a representatividade temática, dispusemos os itens num quadro que designámos de Escala de Avaliação do Debriefing associado à Simulação (EADaS).

O quadro foi antecedido de um conjunto de instruções de preenchimento, com o seguinte teor: "no quadro seguinte são realizadas várias afirmações relativas à discussão que fizemos após a prática simulada (debriefing). Para cada uma, assinale a sua opinião. Não há respostas certas ou erradas. Responda por favor de acordo com a sua verdadeira opinião".

No início do quadro, era ainda apresentada a expressão: "no final do cenário, o debriefing contribuiu para", na continuidade da qual deveria ser lido cada um dos itens.

\section{Validade de conteúdo}

A lista de itens foi avaliada por um painel de peritos que procurou identificar possíveis falhas quanto à clareza das afirmações, a sua representatividade para o constructo e a validade de conteúdo de cada um dos itens, garantindo a validade conceptual.

Do painel de peritos fizeram parte quatro investigadores da Unidade de Investigação em Ciências da Saúde: Enfermagem (UICISA: E), da Escola Superior de Enfermagem de Coimbra (ESEnfC). Dada a simplicidade e clareza do instrumento na sua globalidade, não foi necessário introduzir nenhuma alteração.

\section{Amostra}

A escala foi aplicada a uma amostra acidental de 209 estudantes do $4^{0}$ ano do Curso de Licenciatura em Enfermagem da ESEnfC, em abril de 2012.

A aplicação do questionário aconteceu após nove horas de práticas laboratoriais, por simulação, no final da Unidade Curricular de Enfermagem em Emergências.

Foram incluídos na amostra os participantes que corresponderam aos seguintes critérios de inclusão no estudo (elegibilidade): frequentar a Unidade Curricular de Enfermagem em Emergências; ter estado presente nas aulas da Unidade Curricular de Enfermagem em Emergências onde foram desenvolvidos os cenários e respetivos debriefings; e aceitar participar no estudo.

Responderam ao questionário 209 estudantes, dos quais $81,3 \%$ são mulheres e $18,7 \%$ homens, com uma média de idades de 22 anos.

\section{As experiências clínicas simuladas}

As aulas práticas aconteceram no Centro de Simulação, utilizando como estratégia a resolução de cenários completos em ambiente realista, com dificuldade crescente. Para a resolução dos cenários, os estudantes tiveram à disposição material e equipamento realistas. Foram utilizados simuladores de utentes de média fidelidade (Manequins de Suporte Avançado de Vida Megacod $^{\circledR}$ - adulto, com VitalSim ${ }^{\circledR}$, da Laerdal ${ }^{\circledR}$ ) e de alta-fidelidade (iStan ${ }^{\circledR}$ - adulto, da Meti ${ }^{\circledR}$ ).

Após a resolução de cada cenário, foi conduzido um debriefing estruturado, sempre pelo segundo investigador, seguindo uma estrutura comum: (1) apreciação geral da experiência clínica simulada; (2) balanço relativo às intervenções corretamente desenvolvidas e reforço positivo; (3) balanço relativo às intervenções incorretamente desenvolvidas (ou não desenvolvidas) e respetiva justificação; e (4) pontos-chave relativos à experiência clínica simulada. Cada cenário foi desenvolvido durante cerca de 15 minutos, seguido de outros 15 minutos para o debriefing.

\section{Aspetos formais e éticos}

O estudo insere-se no projeto $A$ simulação no ensino de Enfermagem, inserido na UICISA: E, autorizado 
pela Presidente da ESEnfC e com parecer positivo (P01-09/2010) da Comissão de Ética da Unidade de Investigação supracitada.

A resposta ao questionário foi voluntária, anónima e confidencial. Foi garantido ao estudante a inexistência de quaisquer contrapartidas pela participação ou não participação no estudo, assim como a inexistência de qualquer relação com a avaliação da Unidade Curricular.

Durante todo o processo foram tidos em conta e assegurados os direitos dos participantes à privacidade, ao anonimato, à confidencialidade e à liberdade.

\section{Resultados}

Começámos por analisar a escala, no que se refere à sua confiabilidade global, através da correlação de cada item com o total da escala, o seu efeito sobre o valor de Alpha e as medidas descritivas de resumo.

Obtivemos uma elevada correlação de praticamente todos os itens com o total da escala, o que demonstra o seu bom funcionamento como um todo e que contribui para o elevado valor de Alpha $(0,934)$. Os valores mais baixos de correlação de um item com o global foram de 0,447 e de 0,505 para os itens 41 e 43, respetivamente.

Os valores até aqui encontrados, permitiram-nos avançar para a análise da estrutura da EADaS. Procedemos então à realização de análises fatoriais com rotação ortogonal Varimax com normalização de Kaiser.

A medida de Kaiser-Meyer-Olkin (KMO) é de 0,887, o que significa que existe boa adequação da amostra para análise. $\mathrm{O}$ valor do teste de esfericidade de Bartlett foi de $\chi^{2}=5203,963 ; \mathrm{p}=, 000$, o que nos permitiu prosseguir com a análise fatorial.

Numa primeira tentativa, definindo a extração de valores próprios maiores que um, obtivemos uma solução com cinco fatores, explicando estes 50,391\% da variância. Mas olhando para esta solução, não lhe encontrámos significado racional, para além de apresentar itens a saturar em dois fatores e outros com cargas fatoriais muito baixas.

Procedemos então à análise do Scree Plot e a partir dela ensaiámos uma solução com três fatores.

$\mathrm{Na}$ escolha da solução fatorial final e dos itens que a deveriam integrar, procurámos respeitar os seguintes critérios: (1) a validade convergente do item com o fator - cada item deverá apresentar uma correlação (loading) com o fator $\geq, 30$, e são considerados os fatores com comunalidade $\geq .50$; (2) a validade discriminante do item com o fator - o item deverá estar relacionado apenas com o fator hipotético, devendo obter-se diferença entre cada fator $\geq, 30$; e (3) a solução final encontrada deverá justificar no mínimo 40\% da variância total.

Estes três fatores (dimensões), com valores próprios maiores ou iguais a um, explicam na totalidade $43,99 \%$ da variância total.

Feita a análise e extraídos os componentes, pretendeuse saber que itens constituiriam cada um dos fatores. Seguindo os critérios previamente estabelecidos e as recomendações (Loewenthal, 2001) e sem esquecer o sentido e coerência racional, eliminámos os itens 1, 6, $13,21,23,40$ e 42 por saturarem com valores inferiores a 0,5. Eliminámos também os itens 3, 14, 19, 22, 27, 30, 31,44 e 46, na medida em que a diferença dos valores de saturação entre dois fatores é menor que 0,3.

Apesar dos itens 29, 35 e 38 violarem também o princípio da diferença mínima de 0,3 entre dois fatores, foram mantidos pela sua importância para o constructo teórico.

No final, ficámos com os fatores conforme descrevemos a seguir:

1) O fator 1 explica $16,802 \%$ da variância e nele saturam os itens $25,26,29,33,34,35,37,38,39$, 41, 45, 48 e 49. Estes itens estão todos relacionados com o que concerne aos aspetos psicológicos e sociais inerentes à simulação, pelo que designámos este fator de dimensão valor psicossocial;

2) O fator 2 explica 14,46\% da variância e nele saturam os itens 2, 5, 7, 9, 10, 11, 15, 17 e 18. Estes itens estão sobretudo relacionados com a consolidação de conhecimentos através da discussão, pelo que designámos este fator de dimensão valor cognitivo;

3) $O$ fator 3 explica $12,73 \%$ da variância e nele saturam os itens $4,8,12,16,20,24,28,32,36,43,47$ e 50 . São itens que dizem respeito aos sentimentos ou às afeições. Esta dimensão acolhe os itens formulados em sentido inverso. Designámos este fator de dimensão valor afetivo.

Conforme podemos verificar, esta divisão obtida pela análise fatorial propõe um constructo com significado racional (Tabela 1). Todos os fatores apresentam cargas fatoriais (factorial loadings) elevadas. Repetiuse a análise fatorial, agora apenas com os 34 itens, que renovou esta divisão, confirmando a sua validade de constructo. 
Tabela 1

Matriz de saturação dos itens nos fatores para a solução rodada ortogonal de tipo Varimax com normalização de Kaiser para três fatores $(N=209)$

\begin{tabular}{|c|c|c|c|c|}
\hline \multirow[t]{2}{*}{ Itens } & & \multicolumn{3}{|c|}{ Fatores } \\
\hline & & 1 & 2 & 3 \\
\hline 1 & Refletir sobre o cenário & 194 & 357 & 101 \\
\hline 2 & Estruturar o meu pensamento & 226 &, 566 &, 115 \\
\hline 3 & Consolidar os meus conhecimentos &, 325 &, 519 & ,003 \\
\hline 4 & Me envergonhar frente aos colegas pelos meus erros &, 037 & ,098 &, 664 \\
\hline 5 & Aprender mais &, 234 & 653 &,- 002 \\
\hline 6 & Melhor interagir no e com o grupo &, 417 &, 366 &, 114 \\
\hline 7 & Me focar nos aspetos importantes da atuação &, 271 &, 583 &,- 077 \\
\hline 8 & Me deixar muito ansioso/stressado &,- 029 &, 000 &, 675 \\
\hline 9 & Refletir sobre as minhas competências & ,131 & 709 & ,090 \\
\hline 10 & Identificar prioridades na atuação &, 150 & 670 &, 055 \\
\hline 11 & Melhor identificar os recursos a utilizar na atuação & ,191 & 657 &,- 040 \\
\hline 12 & Me humilhar frente aos outros &, 053 &, 103 &, 754 \\
\hline 13 & Avaliar as minhas próprias capacidades &, 317 & 498 &,- 081 \\
\hline 14 & Identificar as minhas limitações de forma construtiva &, 387 &, 517 &, 070 \\
\hline 15 & Aprofundar conhecimentos específicos relacionados com a atuação &, 305 &, 647 &, 153 \\
\hline 16 & Me deixar em pânico só de pensar em ter de atuar de novo numa situação semelhante & 087 & 049 &, 789 \\
\hline 17 & Identificar aspetos que devo melhorar em atuações futuras &, 254 & 611 &, 117 \\
\hline 18 & Desenvolver competências para a tomada de decisões acertadas & ,224 &, 562 & ,083 \\
\hline 19 & Trocar experiências com os colegas &, 329 &, 525 & ,095 \\
\hline 20 & Criar conflitos no grupo &,- 064 & ,095 &, 585 \\
\hline 21 & Respeitar mais as opiniões dos outros &, 361 &, 254 &,- 059 \\
\hline 22 & Reforçar o espírito de colaboração no grupo &, 536 &, 419 &, 053 \\
\hline 23 & Desenvolver a capacidade de autocrítica &, 348 & 492 &, 185 \\
\hline 24 & Não querer participar em mais nenhuma simulação &,- 037 &, 006 &, 731 \\
\hline 25 & Aumentar a minha autoconfiança &, 626 & ,229 &, 137 \\
\hline 26 & Desenvolver competências de liderança &, 607 &, 250 &,- 093 \\
\hline 27 & Promover o meu autoconhecimento &, 555 &, 455 &,- 028 \\
\hline 28 & Eu me sentir incompreendido &, 028 &, 127 &, 680 \\
\hline 29 & Aumentar o potencial de trabalho em equipa &, 595 &, 353 &, 150 \\
\hline 30 & Melhorar a minha capacidade de adaptação a situações novas &, 587 &, 398 &, 065 \\
\hline 31 & Permitir a aproximação com a realidade &, 525 &, 386 &, 046 \\
\hline 32 & Eu me sentir desrespeitado &, 078 & 081 &, 741 \\
\hline 33 & Eu me sentir realizado &, 637 & ,178 &, 013 \\
\hline 34 & Reforçar a minha iniciativa em situações futuras & ,674 &, 264 &, 132 \\
\hline 35 & Desenvolver a relação de ajuda &, 595 &, 364 &, 012 \\
\hline 36 & Eu sentir que foi uma perda de tempo & ,109 &, 130 & ,695 \\
\hline 37 & Reforçar a minha autonomia para atuar como futuro enfermeiro & ,666 & ,297 &,- 007 \\
\hline 38 & Identificar dificuldades na minha atuação &, 554 &, 311 &, 157 \\
\hline 39 & Promover a autoconsciência (conhecer as próprias emoções) &, 564 & ,237 & ,027 \\
\hline 40 & Eu sentir que nunca serei capaz de fazer as coisas bem numa situação semelhante &, 067 &,- 024 &, 452 \\
\hline 41 & Eu me sentir no centro do processo formativo & ,603 &,- 076 &,- 024 \\
\hline 42 & Relacionar os conhecimentos teóricos e práticos & ,486 &, 323 &, 187 \\
\hline 43 & Eu ter medo de atuar no futuro em situações semelhantes &, 063 &,- 040 &, 588 \\
\hline 44 & Refletir sobre aspetos estruturantes da minha atuação como futuro enfermeiro &, 594 &, 395 &, 134 \\
\hline 45 & Melhorar a minha capacidade de gerir emoções &, 594 & ,293 &, 010 \\
\hline 46 & Me estimular a procurar saber mais sobre os assuntos em causa &, 534 &, 384 &,- 023 \\
\hline 47 & Bloquear o meu raciocínio &, 038 &, 005 &, 787 \\
\hline 48 & Eu sentir orgulho por ser capaz de executar muitas intervenções corretamente & ,695 & ,092 &, 050 \\
\hline 49 & Eu sentir que o professor tem interesse genuíno no meu desenvolvimento profissional &, 517 & 199 &, 097 \\
\hline 50 & Baralhar as minhas ideias a respeito da atuação & 089 & 021 &, 640 \\
\hline
\end{tabular}

VERónica RITA DIAS COUtINHo, et al. $\mid$ Revista de Enfermagem Referência - IV- - n. 2 2-2014 


\section{Fidelidade}

Voltámos a realizar novo cálculo dos valores de Alpha de Cronbach, tendo obtido valores muito bons para o global $(0,899)$ e para cada uma das suas dimensões, nomeadamente: 0,884 para a dimensão psicossocial (13 itens); 0,859 para a dimensão cognitiva (nove itens); e 0,889 para a dimensão afetiva (12 itens). Todos os itens se correlacionam com o global em valores superiores a 0,45 .

\section{Resultados}

Para cada dimensão e global da escala foram somadas as pontuações dos respetivos itens calculando-se o score médio.
Conforme podemos observar nas Tabelas 2, 3 e 4, que resumem a estatística descritiva relativa a cada uma das dimensões da EADaS, verificaram-se diferenças relevantes nos valores de tendência central e dispersão dos vários itens.

$\mathrm{Na}$ dimensão valor psicossocial (Tabela 2), os itens 29, 37 e 38 são os que apresentam valores médios mais elevados e o desvio padrão mais baixos, enquanto os itens 26, 33 e 41 são os que apresentam os valores médios mais baixos com desvio padrão mais elevado.

Em apenas três itens obteve-se avaliação mínima 1 (discordo completamente) e nos restantes existiram respostas com avaliação mínima superior a 1 e máxima 5 (concordo completamente) em todos os itens.

Tabela 2

Estatísticas descritivas dos itens na dimensão valor psicossocial $(n=209)$

\begin{tabular}{|c|c|c|c|c|c|c|c|c|c|c|c|c|c|c|}
\hline \multicolumn{2}{|c|}{ Estatísticas/itens } & 25 & 26 & 29 & 33 & 34 & 35 & 37 & 38 & 39 & 41 & 45 & 48 & 49 \\
\hline \multicolumn{2}{|l|}{ Média } & 4,00 & 3,82 & 4,55 & 3,97 & 4,31 & 4,31 & 4,51 & 4,57 & 4,34 & 3,77 & 4,07 & 4,01 & 4,16 \\
\hline \multicolumn{2}{|l|}{ Mediana } & 4,00 & 4,00 & 5,00 & 4,00 & 4,00 & 4,00 & 5,00 & 5,00 & 4,00 & 4,00 & 4,00 & 4,00 & 4,00 \\
\hline \multicolumn{2}{|l|}{ Moda } & 4,00 & 4,00 & 5,00 & 4,00 & 4,00 & 4,00 & 5,00 & 5,00 & 4,00 & 4,00 & 4,00 & 4,00 & 4,00 \\
\hline \multicolumn{2}{|l|}{ Desvio Padrão } &, 73 &, 83 &, 53 &, 71 &, 60 & ,64 &, 58 &, 54 &, 61 & ,91 &, 77 &, 73 &, 74 \\
\hline \multicolumn{2}{|l|}{ Mínimo } & 2,00 & 1,00 & 3,00 & 3,00 & 2,00 & 2,00 & 2,00 & 3,00 & 2,00 & 1,00 & 2,00 & 2,00 & 1,00 \\
\hline \multicolumn{2}{|l|}{ Máximo } & 5,00 & 5,00 & 5,00 & 5,00 & 5,00 & 5,00 & 5,00 & 5,00 & 5,00 & 5,00 & 5,00 & 5,00 & 5,00 \\
\hline \multirow[t]{3}{*}{ Percentis } & 25 & 4,00 & 3,00 & 4,00 & 3,00 & 4,00 & 4,00 & 4,00 & 4,00 & 4,00 & 3,00 & 4,00 & 4,00 & 4,00 \\
\hline & 50 & 4,00 & 4,00 & 5,00 & 4,00 & 4,00 & 4,00 & 5,00 & 5,00 & 4,00 & 4,00 & 4,00 & 4,00 & 4,00 \\
\hline & 75 & 5,00 & 4,00 & 5,00 & 4,00 & 5,00 & 5,00 & 5,00 & 5,00 & 5,00 & 4,00 & 5,00 & 4,50 & 5,00 \\
\hline
\end{tabular}

Na dimensão valor afetivo (Tabela 3), todos os itens apresentam valores médios superiores a 4 pontos. Os itens 24, 32 e 36 são os que registam valores médios mais elevados e desvio padrão mais baixo, acontecendo o contrário com os itens 8, 16 e 43.
Nesta dimensão obteve-se avaliação mínima 1 (discordo completamente) em quatro itens e nos restantes existiram respostas com avaliação mínima superiora 1 e máxima de 5 (concordo completamente) em todos os itens.

Tabela 3

Estatísticas descritivas dos itens da dimensão valor afetivo $(N=209)$

\begin{tabular}{|c|c|c|c|c|c|c|c|c|c|c|c|c|c|}
\hline Estatísticas/itens & & 4 & 8 & 12 & 16 & 20 & 24 & 28 & 32 & 36 & 43 & 47 & 50 \\
\hline Média & & 4,61 & 4,04 & 4,78 & 4,53 & 4,73 & 4,83 & 4,73 & 4,81 & 4,89 & 4,48 & 4,55 & 4,55 \\
\hline Mediana & & 5,00 & 4,00 & 5,00 & 5,00 & 5,00 & 5,00 & 5,00 & 5,00 & 5,00 & 5,00 & 5,00 & 5,00 \\
\hline Moda & & 5,00 & 5,00 & 5,00 & 5,00 & 5,00 & 5,00 & 5,00 & 5,00 & 5,00 & 5,00 & 5,00 & 5,00 \\
\hline Desvio Padrão & &, 72 & 1,06 &, 54 &, 73 &, 67 &, 46 &, 56 &, 50 &, 35 &, 89 &, 71 &, 84 \\
\hline Mínimo & & 2,00 & 1,00 & 2,00 & 2,00 & 1,00 & 2,00 & 2,00 & 2,00 & 3,00 & 1,00 & 2,00 & 1,00 \\
\hline Máximo & & 5,00 & 5,00 & 5,00 & 5,00 & 5,00 & 5,00 & 5,00 & 5,00 & 5,00 & 5,00 & 5,00 & 5,00 \\
\hline \multirow[t]{3}{*}{ Percentis } & 25 & 4,00 & 3,50 & 5,00 & 4,00 & 5,00 & 5,00 & 5,00 & 5,00 & 5,00 & 4,00 & 4,00 & 4,00 \\
\hline & 50 & 5,00 & 4,00 & 5,00 & 5,00 & 5,00 & 5,00 & 5,00 & 5,00 & 5,00 & 5,00 & 5,00 & 5,00 \\
\hline & 75 & 5,00 & 5,00 & 5,00 & 5,00 & 5,00 & 5,00 & 5,00 & 5,00 & 5,00 & 5,00 & 5,00 & 5,00 \\
\hline
\end{tabular}


Na dimensão valor cognitivo (Tabela 4), todos os itens apresentam valores médios superiores a 4 pontos e mais de metade das respostas com a pontuação máxima (concordo completamente). 0 item 9 é o que apresenta menor valor médio e desvio padrão mais elevado, sendo os itens 2, 10 e 17 os que apresentam valores médios mais elevados, com desvio padrão mais baixo. Em apenas um item se obteve avaliação mínima 1 (discordo completamente) e nos restantes existiram respostas com avaliação mínima superior a 1 e máxima 5 (concordo completamente) em todos os itens.

Tabela 4

Estatísticas descritivas dos itens da dimensão valor cognitivo $(N=209)$

\begin{tabular}{|c|c|c|c|c|c|c|c|c|c|c|}
\hline Estatísticas/itens & & 2 & 5 & 7 & 9 & 10 & 11 & 15 & 17 & 18 \\
\hline Média & & 4,70 & 4,63 & 4,57 & 4,40 & 4,66 & 4,51 & 4,54 & 4,66 & 4,56 \\
\hline Mediana & & 5,00 & 5,00 & 5,00 & 4,00 & 5,00 & 5,00 & 5,00 & 5,00 & 5,00 \\
\hline Moda & & 5,00 & 5,00 & 5,00 & 4,00 & 5,00 & 5,00 & 5,00 & 5,00 & 5,00 \\
\hline Desvio Padrão & &, 46 & ,49 &, 54 &, 59 &, 51 &, 56 &, 52 &, 49 &, 57 \\
\hline Mínimo & & 3,00 & 3,00 & 2,00 & 2,00 & 2,00 & 3,00 & 3,00 & 3,00 & 1,00 \\
\hline Máximo & & 5,00 & 5,00 & 5,00 & 5,00 & 5,00 & 5,00 & 5,00 & 5,00 & 5,00 \\
\hline \multirow[t]{3}{*}{ Percentis } & 25 & 4,00 & 4,00 & 4,00 & 4,00 & 4,00 & 4,00 & 4,00 & 4,00 & 4,00 \\
\hline & 50 & 5,00 & 5,00 & 5,00 & 4,00 & 5,00 & 5,00 & 5,00 & 5,00 & 5,00 \\
\hline & 75 & 5,00 & 5,00 & 5,00 & 5,00 & 5,00 & 5,00 & 5,00 & 5,00 & 5,00 \\
\hline
\end{tabular}

Na Tabela 5, podemos observar alguns valores estatísticos descritivos relativos à globalidade da EADaS e a cada uma das suas dimensões. A média dos itens para a dimensão valor psicossocial é de 4,27 pontos, com um desvio padrão de 0,41 pontos, enquanto a dimensão valor afetivo apresenta uma média de itens ligeiramente superior (4,62 pontos) e um desvio padrão de 0,46 pontos. Já a dimensão valor cognitivo apresenta uma média dos itens de 4,56 pontos, com um desvio padrão de 0,34 pontos. Globalmente, a média é de 4,48 pontos e o desvio padrão de 0,30 pontos.

Tabela 5

Estatísticas descritivas relativas à EADaS $(N=209)$

\begin{tabular}{|c|c|c|c|c|}
\hline Estatísticas/EADaS & $\begin{array}{c}\text { Dimensão valor } \\
\text { psicossocial }\end{array}$ & $\begin{array}{l}\text { Dimensão valor } \\
\text { afetivo }\end{array}$ & Dimensão valor cognitivo & Global \\
\hline Média & 4,27 & 4,62 & 4,56 & 4,48 \\
\hline Mediana & 4,27 & 4,76 & 4,60 & 4,51 \\
\hline Moda & 3,95 & 5,00 & 5,00 & 5,00 \\
\hline Desvio Padrão &, 41 &, 46 &, 34 &, 30 \\
\hline Variância &, 17 & ,21 & ,12 & ,09 \\
\hline Mínimo & 2,82 & 2,69 & 3,80 & 3,37 \\
\hline Máximo & 5,00 & 5,00 & 5,00 & 5,00 \\
\hline Percentis 25 & 3,95 & 4,44 & 4,26 & 4,28 \\
\hline 50 & 4,27 & 4,76 & 4,60 & 4,51 \\
\hline 75 & 4,59 & 5,00 & 4,90 & 4,72 \\
\hline
\end{tabular}

Analisámos a distribuição dos valores da $\mathrm{EADaS}$ no seu global e para cada uma das dimensões, tendo para isso realizado o teste de Kolmogorov-Smirnov com correção de Lilliefors. Os valores obtidos revelam que a distribuição não é normal tanto para a dimensão cognitiva como para a dimensão afetiva ( $p$ $<, 05$ ), pelo que nos testes seguintes foram utilizadas medidas não paramétricas.
Realizámos o teste U de Mann-Whitney, no sentido de avaliar se a EADaS estava a distinguir o valor atribuído ao debriefing entre homens e mulheres, verificando que as pequenas diferenças que existem não são estatisticamente significativas.

De seguida, realizámos um conjunto de testes de significância de correlação de Spearman (Tabela 6), procurando relações entre as diferentes dimensões 
e o global da EADaS. Encontrámos cada uma das dimensões fortemente correlacionadas com 0 global, sendo essas correlações estatisticamente significativas. Existe uma correlaçãa forte entre a dimensão valor psicossocial e a dimensão valor cognitivo e uma correlação fraca entre a dimensão valor afetivo e a dimensão valor cognitivo, sendo ambas estatisticamente significativas. Entre a dimensão valor afetivo e a de valor psicossocial a correlação é fraca e não significativa.

Tabela 6

Resultados do Teste de Correlação de Spearman entre as dimensões e a globalidade da EADaS ( $N=209)$

\begin{tabular}{llccc}
\hline & & $\begin{array}{c}\text { Dimensão } \\
\text { valor psicossocial }\end{array}$ & $\begin{array}{c}\text { Dimensão } \\
\text { valor cognitivo }\end{array}$ & $\begin{array}{c}\text { Dimensão } \\
\text { valor afetivo }\end{array}$ \\
\hline Dimensão cognitiva & $\mathrm{r}_{\mathrm{s}}$ &, $757^{* *}$ & & \\
& Sig. (2-tailed) &, 000 &, $167^{*}$ & \\
Dimensão afetiva & $\mathrm{r}_{\mathrm{s}}$ &, 135 &, 017 &, $638^{* *}$ \\
& Sig. (2-tailed) &, 057 &, $805^{* *}$ &, 000 \\
\hline
\end{tabular}

* Correlação significativa para $p<0,05$; **Correlação significativa para $p<0,01$

\section{Discussão}

A avaliação dos resultados não pode deixar de ter em conta algumas limitações do estudo, impondose prudência na sua interpretação e generalização. A especificidade da amostra é uma das limitações. A inexistência de instrumentos de avaliação de debriefing, se por um lado contribui para justificar a pertinência da construção e validação da escala, vem por outro lado impossibilitar o estudo da validade concorrente.

Já o percurso de desenvolvimento e validação da EADaS, parece-nos ser um ponto forte que vem contribuir para a validação científica dos resultados que aqui apresentamos, que são indicadores do potencial de utilização da escala em investigações futuras.

O valor de Alpha de Cronbach da escala final, com 34 itens, foi de 0,899 , o que revela boa consistência interna. A análise fatorial, seguida de rotação varimax extraiu três dimensões. A composição das dimensões sugere os significados: valor psicossocial, valor cognitivo e valor afetivo.

A simulação tem sido utilizada para melhorar o desempenho dos estudantes nas competências, alterar atitudes e aumentar o conhecimento (Jeffries \& Rizzolo, 2006; Lasater, 2007). O debriefing é um elemento essencial na aprendizagem por simulação, proporcionando aprendizagens ativas, que permitem aos estudantes vivenciar situações clínicas e fazer uso das habilidades cognitivas, afetivas e psicomotoras (Childs \& Sepples, 2006), o que vai ao encontro dos nossos resultados.

Podendo variar as respostas com avaliação mínima (1) e avaliação máxima (5), os estudantes consideram em percentagens elevadas que o debriefing contribuiu para estruturar o pensamento (99,5\%); identificar prioridades na atuação $(99,1 \%)$; identificar aspetos que devem melhorar em atuações futuras (99,1\%); identificar dificuldades na atuação (97,6\%); aumentar o potencial de trabalho em equipa (98,1\%); reforçar a autonomia para atuar como futuros enfermeiros (96,6\%), corroborando com o que Hodges (2006) e Dieckmann, Manser, Wehner, e Marcus (2007) referem. Estes autores defendem que o debriefing é importante para maximizar a aprendizagem e facilitar a mudança tanto a nível individual como sistemático. Em contrapartida, no nosso estudo, os estudantes referem que o debriefing não foi uma perda de tempo e foi um momento em que se sentiram respeitados, indo ao encontro do que Lasater (2007) refere, assim como Jeffries e Rizzolo (2006), em que a participação ativa e a oportunidade de aplicar a avaliação, observação e habilidades para resolver problemas, seguido por uma experiência de reflexão, leva ao aumento da autoconfiança dos alunos.

Segundo Rodrigues e Baía (2012), "A função do educador moderno implica cada vez mais o desenvolvimento de competências para agir em mediação pedagógica, orientando a ação em 
compromissos recíprocos e justos para ambas as partes" (p. 201). Ao educador é solicitada a força e ética para ajudar os seus orientandos a tornarem-se autores dos próprios percursos, a tomar decisões, resolver problemas, enfrentar a dúvida e o risco, e sobretudo a serem proactivos na busca das melhores experiências de desenvolvimento e aprendizagem.

O debriefing proporciona aos estudantes a oportunidade de refletirem sobre as suas aprendizagens durante a prática simulada e de definirem como podem ter um desempenho diferente numa situação similar. Por outro lado, o debriefing também oferece aos estudantes uma realidade, uma forma de se verem através dos olhos do docente e dos seus pares, em consonância com o descrito por Overstreet (2009). Os nossos estudantes partilham destas mesmas ideias, visto que 98,1\% refere ter desenvolvido competências para a tomada de decisão acertada; 76,6\% refere que aumentou a autoconfiança; e $84,2 \%$ sentiu que o professor tem interesse genuíno no seu desenvolvimento profissional.

Em termos gerais, têm sido vários os autores a afirmar os efeitos benéficos do debriefing, tais como: Jeffries, 2007; Kardong-Edgren et al., 2008; Campbell e Daley, 2009; Overstreet, 2009; Dreifuerst, 2009; entre outros. O presente estudo vem confirmar estas conclusões e concretizar algumas áreas específicas em que se comprova o impacto positivo do debriefing no estudante, conjugado com a prática simulada.

Tal como analisado, o debriefing foi bem valorizado pelos estudantes, verificando-se que a dimensão valor afetivo tem a média mais alta em relação às outras, embora com a correlação mais baixa no seu geral, ainda que estatisticamente significativa. Arriscamos assim dizer que o debriefing traduz uma estratégia que proporciona aos estudantes uma relação afetiva entendida pelos mesmos como forma de proteção.

\section{Conclusão}

O debriefing é uma parte fundamental do ensino por simulação. Utiliza a reflexão estruturada como forma de consolidar os conhecimentos de forma significativa para os estudantes, olhando o que se fez e procurando as justificações e os mecanismos mentais associados à decisão. Este é um processo central na formação de enfermeiros, pois não se pretendem profissionais que apenas saibam fazer coisas, mas que saibam como, quando e porquê as fazer, assim como opções para fazer de forma diferente, sempre que tal se mostre necessário.

Não tendo encontrado na literatura nenhum instrumento que permitisse avaliar o impacto do debriefing estruturado no estudante, associado à prática simulada, entendendo que esse seria um aspeto central, propusemo-nos à concretização desse objetivo: criar e validar um instrumento destinado a avaliar o impacto do debriefing estruturado nos estudantes, associado à prática simulada.

Apesar das limitações, foi possível construir uma escala que permite avaliar o impacto do debriefing estruturado nos estudantes associado a práticas simuladas, apresentando esta escala boas propriedades psicométricas, perspetivando assim o seu potencial de utilização em investigação científica. O resultado final foi uma escala com 34 itens divididos por três dimensões: a dimensão valor psicossocial, a dimensão valor cognitivo e a dimensão valor afetivo. Estas três dimensões foram obtidas por análise fatorial. No seu conjunto, a escala apresenta muito boas propriedades psicométricas com valores de Alpha superiores a 0,80 no seu global e em cada uma das dimensões.

O nosso estudo vem demonstrar que a escala poderá afigurar-se como uma ferramenta útil para avaliar o valor atribuído ao debriefing associado à simulação em estudantes de Enfermagem, acreditando os autores que o seu valor sairá reforçado em investigações futuras e contribuirá para a melhoria das práticas dos enfermeiros e prestação de cuidados de Enfermagem de maior qualidade.

Assim, sugerimos que sejam realizados mais estudos em Portugal sobre o debriefing, que possam aplicar esta escala, e que sejam divulgados os resultados aos docentes e outros profissionais de forma a poder ser uma mais-valia na definição conjunta de estratégias pedagógicas a utilizar nas unidades curriculares.

\section{Referências bibliográficas}

Baldwin, K. (2007). Friday night in the pediatric emergency department: A simulated exercise to promote clinical reasoning in the classroom. Nurse Educator, 32(1), 24-29.

Campbell, S., \& Daley, K. (2009). Simulation scenarios for nurse educators: Making it real. New York, NY: Springer. 
Childs, J., \& Sepples, S. (2006). Cllinical teaching by simulation: Lessons learned from a complex patient care scenario. Nusing Education Perspectives, 27(3), 154-158.

Dieckmann, P., Manser, T., Wehner, T., \& Marcus, R. (2007). Reality and fiction cues in medical patient simulation. An interview study with anesthesiologists. Journal of Cognitive Engineering and Decision Making, 1(2), 148-168.

Dreifuerst, K. (2009). The essential of debriefing in simulation learning: A concept analysis. Nursing Education Perspectives, 30(2), 109-114.

Gaba, D., Howard, S., Fish, K., Smith, B., \& Sowb, Y. (2001). Simulation-based training in anesthesia crisis resource management (ACRM): A decade of experience. Simulation e Gaming, 32(2), 175-193.

Gururaja, R., Yang, T., Paige, J., \& Chauvin, S. (2008). Examining the effectiveness of debriefing at the point of care in simulation-based operating room team training. In $\mathrm{K}$. Henriksen, J. Battles, M. Keyes, \& M. Grady, Advances in patient safety: New directions and alternative approaches (Vol. 3). Rockville, MD: Agency for the healthcare research and Quality. Recuperado de http://www.ncbi.nlm.nih.gov/ books/NBK43676/

Heukelom, V., Begaz, T., \& Treat, R. (2010). Comparison of postsimulation debriefing versus in simulation debriefing $\mathrm{i}$ medical simulation. Simulation in Healthcare: Journal of the Society for Simulation in Helthcare, 5(2), 91-97.

Hodges, B. (2006). Medical education and the maintenance of incompetence. Medical Teacher, 28(8), 690-696.

Jeffries, P. (2007). Simulation in nursing education:from conceptualization to evaluation. New York, NY: National League for Nursing.

Jeffries, P., \& Rizzolo, A. (2006). Designing and implementing models for the innovative use of simulation to teach nursing care of ill adults and children: A National, multi-site, multimethod study. In P. Jeffries (Ed.), Simulation in nursing education: From conceptualization to evaluation. (pp.147159). New York, NY: National League for Nursing.

Kardong-Edgren, S., Starkweather, A., \& Ward, L. (2008). The integration of simulation into a clinical foundations of nursing course: Student and faculty perspectives. International Journal of Nursing Education Scholarship, 5(1),1-16.

Lasater, K. (2007). Clinical judgment development: Using simulation to create an assessment rubric. Journal of Nursing Education, 46(11), 496-503.

Loewenthal, K. (2001). An introduction to psychological tests and scales. Hove, Reino Unido: Psychological Press.

Martins, J. (2009). Atuação do enfermeiro no sector de urgencias: Gestão para o desenvolvimento de competencias. In W. Malagutti \& K. Cardoso, Gestão do serviço de enfermagem no mundo globalizado (pp. 175-189). Rio de Janeiro, Brasil: Rubio.

Martins, J., Mazzo, A., Baptista, R., Coutinho, V., Gody, S., Mendes, I., \& Trevizan, M. (2012). The simulated clinical experience in nursing education: A historical review. Acta Paulista de Enfermagem, 25(4), 619-625.

Overstreet, M. (2009). The current practice of nursing clinical simulation debriefing: A multiple case study (Tese de Doutoramento). Recuperado de http://trace.tennessee.edu/ cgi $/$ viewcontent.cgi?article $=1696 \&$ context $=u t k \_$graddiss

Rodrigues, M., \& Baia, M. ( 2012). Mediação e acompanhamento na formação, educação e desenvolvimento profissional. Revista de Enfermagem Referência, 3(7),199-205.

Shinnick, M., Horwich, T., \& Steadman, R. (2011). Debriefing: The most importante component in simulation? Clinical Simulation in Nursing, 7(3),105-111.

Warrick, D., Hunsaker, P., Cook, C., \& Altman, S. (1979). Debriefing experiential learning exercises. Journal of Experiential Learning and Simulation, 1(2), 91-100. 